\title{
APLICAÇÃO DO MÉTODO DE IRRADIAÇÃO TRIDIMENSIONAL NO MONITORAMENTO DE BARRAGENS
}

Application of the three-dimensional radiation method for monitoring dams

\author{
ALYNE RAMINELLI SIGUEL ${ }^{1}$ \\ PEDRO LUIS FAGGION ${ }^{2}$ \\ LUÍS AUGUSTO KOENIG VEIGA ${ }^{2}$ \\ CARLOS AURÉLIO NADAL ${ }^{2}$ \\ MARCELO DA SILVA MATTOS ${ }^{2}$ \\ MARCOS ALBERTO SOARES ${ }^{3}$ \\ ${ }^{1}$ Universidade Tecnológica Federal do Paraná - UTFPR \\ Curitiba - Paraná - Brasil \\ ${ }^{2}$ Universidade Federal do Paraná - UFPR \\ Curitiba - Paraná - Brasil \\ ${ }^{3}$ Compainha Paranaense de Energia Elétrica- COPEL \\ Curitiba - Paraná - Brasil \\ alynesiguel@utfpr.edu.br; faggion@ufpr.br; kngveiga@ufpr.br; cnadal@ufpr.br; \\ marcelo@areaexata.com.br; marcos.asoares@copel.com.
}

\section{RESUMO}

Com a finalidade de verificar a estabilidade de pontos de monitoramento previamente selecionados e materializados a jusante da barragem da usina hidrelétrica Mauá, localizada no rio Tibagi, entre os municípios de Ortigueira e Telêmaco Borba, estado do Paraná, aplicou-se o método de irradiação topográfica tridimensional para determinar suas coordenadas. O estudo do comportamento se dá pela comparação das coordenadas dos pontos obtidas entre duas ou mais épocas distintas. Para isso, estabeleceu-se uma rede geodésica materializada por meio de pilares de concreto, dotados de um sistema de centragem forçada e construídos de forma que a fundação alcançasse a rocha da região. Estes, foram monitorados através de técnicas GNSS onde constatou-se sua estabilidade, servindo então como referência para o monitoramento dos pontos irradiados. Após distintas campanhas 
de levantamentos, realizadas antes e durante a formação do reservatório, utilizou-se um aplicativo em ambiente MatLab, desenvolvido para as finalidades deste projeto, onde são feitas análises e correções nos dados obtidos. Posteriormente, são calculadas as coordenadas tridimensionais dos pontos monitorados. Como resultados apresentam-se a variação das coordenadas utilizando-se a primeira campanha de levantamento, efetuada antes do início da formação do reservatório, como referência.

Palavras-chave: Monitoramento de Estruturas; Levantamentos Geodésicos; Irradiação Tridimensional.

\section{ABSTRACT}

In order to check the stability of monitored points previously selected and materialized downstream of the Mauá hydroelectric plant dam, located in Tibagi river, between the cities of Ortigueira and Telêmaco Borba, state of Paraná, the 3D irradiation topographic method was held to determine the coordinates of these points. The study of the monitored point behavior is given by the comparison of their coordinates, between two or more distinct epochs. For this, it was established a geodetic network materialized by concrete pillars, equipped with a system of forced centering and constructed so that the foundation reached the rocks of the region. These were monitored using GNSS techniques where it was found stability, then serving as reference for monitoring points irradiated. After different surveys campaigns carried out before and during the formation of the reservoir, it was used an application in MatLab environment, developed for the purpose of this project, where analyses and corrections were done in the data obtained. Thereafter, the three-dimensional coordinates from monitored points were calculated. The results show the variation of the coordinates using the first campaign of surveying, made before the reservoir formation, as a reference.

Keywords: Monitoring Structures; Geodesic Surveys; Three-dimensional Irradiation.

\section{INTRODUÇÃO}

A instrumentação e o estudo do comportamento de obras de engenharia possuem relevância nos dias atuais, pois tem como finalidade a observação destes empreendimentos. Neste contexto, estão inclusas as barragens, obras que geralmente são associadas a um elevado potencial de risco devido à possibilidade de um eventual colapso, desta forma, toda barragem deve ser instrumentada de acordo com seu porte e riscos associados (BRASIL, 2002).

A construção e operação de usinas hidrelétricas estão relacionadas com importantes benefícios econômicos, pois a energia gerada é um dos fatores determinantes a diversas ações humanas. Devido a este fato, em muitos países existem legislações que regulamentam o controle e a segurança de barragens com base em regras específicas (CASTRO e HENRIQUES, 2008). No Brasil, segundo a 
Eletrobrás, deve ser previsto um sistema de instrumentação com vistas à auscultação do comportamento dessas estruturas para as fases de construção, do enchimento do reservatório e da operação.

Neste artigo apresenta-se o processo aplicado para o monitoramento tridimensional de alvos engastados na face de jusante da barragem da usina hidrelétrica Mauá. Neste contexto, serão analisadas as coordenadas obtidas entre campanhas distintas, antes e durante a formação do reservatório, indicando se houve ou não a movimentação dos pontos previamente selecionados.

A metodologia adotada pode servir como referência ao monitoramento de outros empreendimentos. Para isso, empregou-se o método de irradiação 3D, onde as observações são realizadas com o auxílio de uma estação total robotizada. Os dados obtidos permitem a formação de um conjunto adicional de elementos que servirão ao gerenciamento e controle da segurança da usina, devendo estes ser analisados juntamente com outras informações pela empresa responsável.

\section{MONITORAMENTO GEODÉSICO}

O Departament of The Army (1994), dos Estados Unidos, classifica as técnicas de medição e instrumentação para o monitoramento geométrico das deformações de estruturas em dois grupos: os levantamentos geodésicos que incluem os levantamentos terrestres, posicionamento por satélites, fotogramétrico e algumas técnicas especiais (interferometria, nivelamento hidrostático e outros); e as medidas geotécnicas e estruturais de deformações locais, utilizando extensômetros, inclinômetros, fios de prumo, etc. O método geodésico é muito útil e fornece uma visão global da deformação. Enquanto os métodos não geodésicos são benéficos para prover medidas de pontos específicos (CHRZANOWSKI, 1986, apud SETAN et al., 2003).

No âmbito dos levantamentos geodésicos alguns métodos são utilizados para o monitoramento de grandes estruturas, entre eles estão o nivelamento geométrico de primeira ordem, levantamento gravimétrico, posicionamento GNSS, irradiações, triangulações e trilaterações. Adiante será descrita a metodologia empregada no monitoramento da barragem da UHE Mauá. Esta, localiza-se no rio Tibagi, entre os municípios de Ortigueira e Telêmaco Borba, no estado do Paraná. A barragem fica na região do Salto Mauá, 600 metros a montante da já existente Usina Presidente Vargas e permite a formação de um reservatório com quase $84 \mathrm{~km}^{2}$ de superfície (CONSÓRCIO ENERGÉTICO CRUZEIRO DO SUL, 2012).

\subsection{Irradiação Tridimensional}

A determinação das coordenadas tridimensionais de um ponto pode ser obtida através do método de irradiação 3D ou também chamado em algumas literaturas de polar. Este, baseia-se na medida de direções horizontais, ângulos verticais e distâncias dos pontos de interesse em relação a uma direção conhecida, tomada como referência. 
A representação das coordenadas é feita através de um sistema com três eixos ortogonais entre si, que formam um sistema de coordenadas tridimensionais ortogonais. Logo, para que um ponto seja estabelecido de forma única é necessário determinar suas coordenadas cartesianas ortogonais $\mathrm{X}, \mathrm{Y}$ e Z $\mathrm{Z}$, onde $\mathrm{X}$ é denominado de abscissa, Y de ordenada e Z de cota (MIRANDA et al., 2009).

Nos sistemas de coordenadas cartesianas tridimensionais trabalha-se com coordenadas polares (MIRANDA et al., 2009). Estas são obtidas a partir de: direções horizontal $(\alpha)$ medido no plano XY, ângulo zenital $(Z)$ medido no plano vertical que contém o eixo $\mathrm{Z}$ e o ponto observado, e a distância inclinada (Di) medida do equipamento ao ponto considerado (figura 1).

Figura 1 - Esquema representativo do método polar. Fonte: A autora (2013).

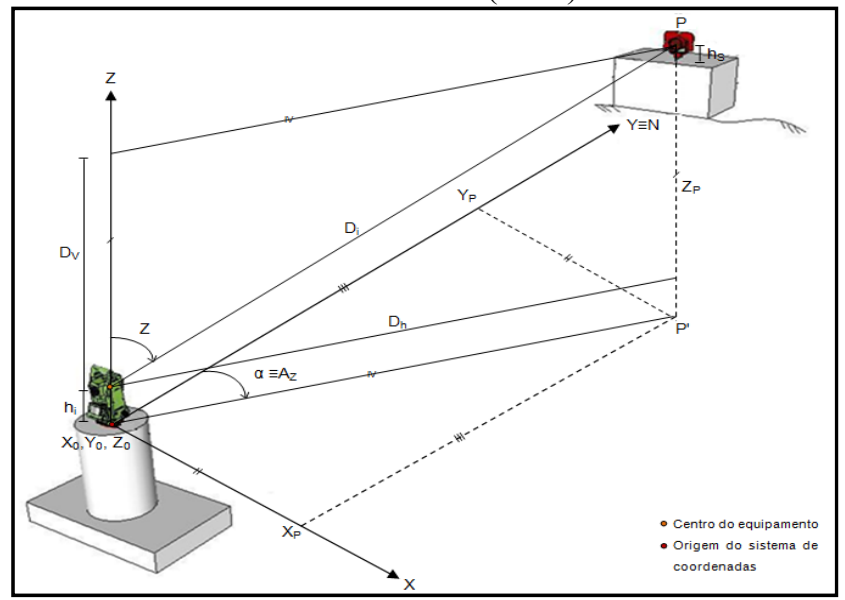

As coordenadas cartesianas tridimensionais dos pontos de interesse são obtidas através das Equações 1, 2 e 3:

$$
\begin{aligned}
& X_{P}=X_{0}+D_{i} \operatorname{sen} Z \cdot \operatorname{sen} A_{z} \\
& Y_{P}=Y_{0}+D_{i} \operatorname{sen} Z \cdot \cos A_{z} \\
& Z_{P}=Z_{0}+\left(h_{i}-h_{s}+D_{i} \cos Z\right)
\end{aligned}
$$

onde:

$X_{p}, Y_{p}, Z_{P}$ - Coordenadas do ponto $\mathrm{P}$ no referencial local;

$X_{0}, Y_{0}, Z_{0}$ - Coordenadas da origem;

$h_{i}$ - Altura do instrumento; 
$h_{s}$ - Altura do sinal;

$D_{i}$ - Distância inclinada;

$A_{z}$ - Azimute (considerando que o eixo Y esta orientado para a direção norte); e $Z$ - Ângulo zenital.

\subsection{Rede Geodésica de Monitoramento}

De acordo com Departament of the Army (1994), dos Estados Unidos, os procedimentos gerais para monitorar a deformação de uma estrutura envolvem a medida de deslocamentos espaciais de pontos selecionados (alvos), a partir de pontos de referência, que têm suas posições controladas. Estes pontos de referência, na UHE Mauá, são definidos por meio de uma rede geodésica de monitoramento. Esta, é composta por dois pilares geodésicos a montante (PG01 e PG02), um ponto localizado na crista da barragem (CG01) e um pilar a jusante (PG03) (figura 2).

Figura 2 - Posições aproximadas dos pilares que formam a rede geodésica de monitoramento da UHE Mauá.

Fonte: A autora (2013).

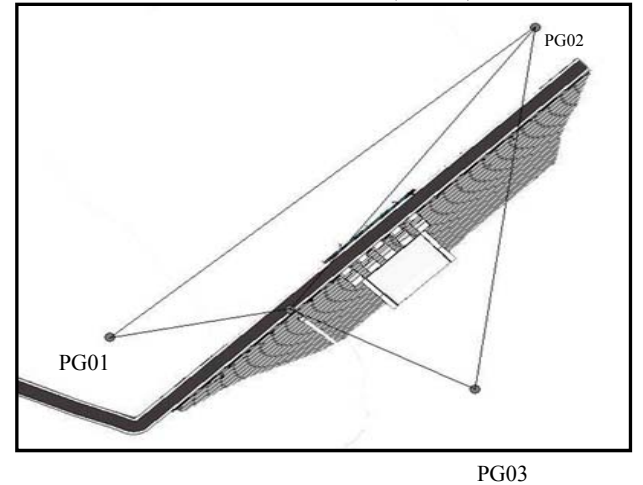

Após diversas campanhas GNSS realizadas antes e durante o enchimento do reservatório, ocorrido em 28 de junho de 2012, foi possível verificar que as coordenadas determinadas não sofreram variações significativas, por isso, adotou-se como referência as coordenadas dos pilares obtidas na primeira campanha de levantamento, realizada antes do início da formação do reservatório. Estas coordenadas foram transformadas em topográficas locais e utilizadas no cálculo das coordenadas dos pontos de monitoramento.

\subsubsection{Pilares da Rede Geodésica de Monitoramento}

Os três pilares que compõem a rede geodésica de monitoramento constituem pontos fixos que servem como referência para as observações efetuadas. Para 
garantir a estabilidade estes foram construídos de forma que a fundação alcançasse as rochas da região (figura 3 ).

Figura 3 - Esquema construtivo dos pilares geodésicos.

Fonte: A autora (2013).

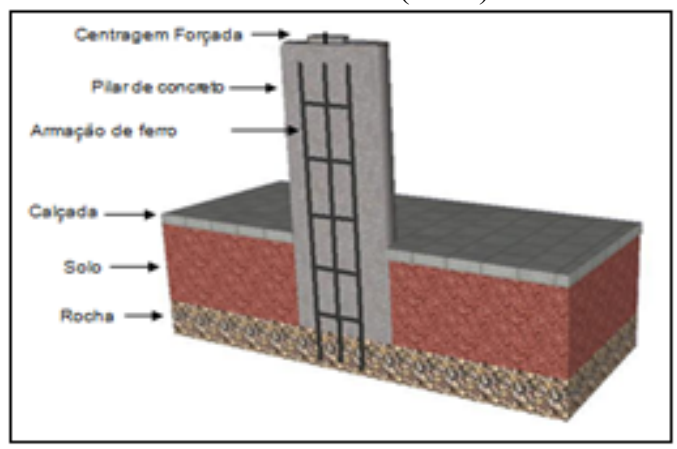

Todos os pilares são dotados de um sistema de centragem forçada que serve para materializar os pontos de referência. Este sistema consiste em uma placa circular de alumínio que é fixada sobre o pilar por meio de parafusos. No centro desta placa há uma rosca que permite a fixação do instrumento utilizado para as observações, garantindo a reocupação das mesmas posições em épocas distintas de monitoramento. Segundo Nadal et al., (2000), a estimativa da repetibilidade na reocupação é da ordem do décimo de milímetro.

\subsubsection{Manutenção da Altura dos Conjuntos de Bases e Prismas e da Estação Total:}

Em alguns dos pontos dotados do sistema de centragem forçada (PG03 e PG02) foi instalada a estação total, utilizada para o monitoramento, e um conjunto de base e prisma que servirão para a orientação do equipamento no momento das irradiações. As alturas da base do prisma e da estação total influenciam diretamente nas medidas dos ângulos verticais e das distâncias inclinadas, e como as coordenadas planialtimétricas $(\mathrm{X}, \mathrm{Y}, \mathrm{Z})$ dos pontos observados são dependentes destas observações, definiu-se estabelecer um procedimento para fixar tais alturas. Manter a altura do instrumento e das bases fixas e conhecidas com precisão a priori é importante para as medidas (angulares e lineares) realizadas. Desta forma, eliminam-se a necessidade da medida em campo destas grandezas, evitando-se erros e viabilizando a comparação entre as coordenadas obtidas nas diferentes campanhas.

No processo convencional de nivelamento das bases são utilizados três parafusos calantes, desta forma, a cada posicionamento um novo plano horizontal é definido. Logo, o problema principal é conhecer a altura do instrumento e das bases e prismas com precisão suficiente para as operações de monitoramento. Daí surge à necessidade de determinar estas quantidades em laboratório, estabelecendo também 
um procedimento que mantenha a altura de cada uma das bases ao longo dos levantamentos.

Visando solucionar este impasse desenvolveu-se uma peça que permite travar um dos parafusos calantes (figura 4). Assim, a altura da base se mantém constante, pois ao realizar o nivelamento com apenas dois parafusos a altura do plano horizontal será definida em função da posição do calante que está fixo.

Figura 4 - Peça utilizada para travar o parafuso calante.

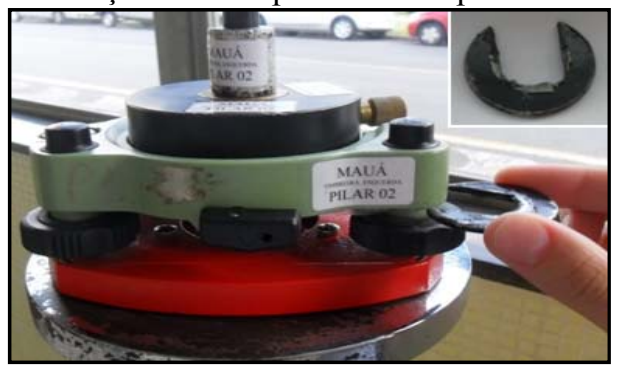

Na figura 5 (a) e (b) é possível visualizar um dos parafusos calantes travado (A) com a base desnivelada. Neste caso, o ponto A pertence ao plano $\pi$ ' e $\pi$ (plano horizontal), enquanto os parafusos B e C pertencem ao plano $\pi$ '. Já na Figura 5 (c) há a indicação da base nivelada com todos os pontos pertencendo ao mesmo plano.

Figura 5 - Sistema de manutenção da altura das Bases Nivelantes.

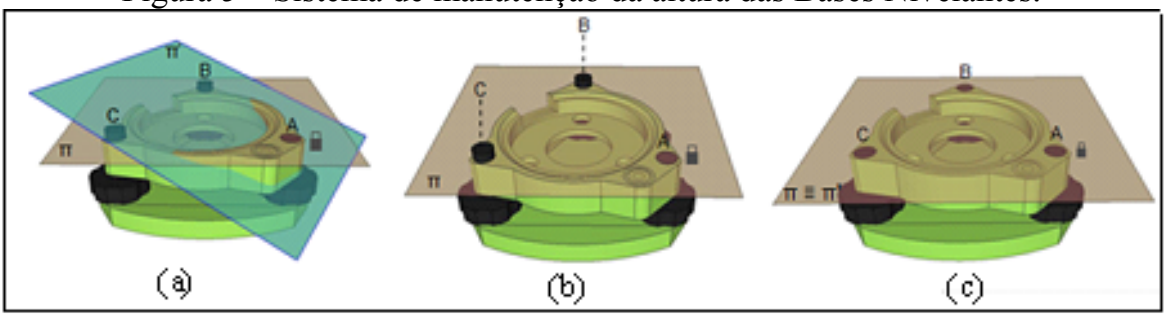

Descrição da figura 5:

a) base nivelante fora de nível e os planos $\pi$ e $\pi$ ';

b) base nivelante fora de nível e o plano $\pi$;

c) base nivelada e os pontos a, b e c pertencentes ao plano $\pi$.

Para manter a altura do conjunto de bases e prismas ao longo das diversas campanhas de levantamentos e também para medir estas alturas, foram efetuados os seguintes procedimentos:

1) Primeiramente ajustaram-se os parafusos calantes das bases de forma que todos ficassem em uma mesma altura média. Em seguida, foram encaixadas as peças 
desenvolvidas;

2) Como a altura das bases são aproximadamente iguais tomou-se uma delas como referência e mediu-se sua altura utilizando um paquímetro. A altura das demais foi ajustada apertando-se ou soltando o parafuso calante "travado";

3) Em seguida, encaixou-se uma abraçadeira ao redor do parafuso fixo impedindo o acesso ao mesmo;

4) Por fim, foram encaixados os prismas sobre as bases e medidas as alturas de todos os conjuntos (base e prisma) e da estação total, através de técnicas de nivelamento de precisão, utilizado um conjunto formado por uma mira de invar e um nível digital.

- As alturas medidas foram:

- Conjunto 1: 176,9 mm;

- Conjunto 2: 177,3 mm;

- Conjunto 3: 176,7 mm;

- Conjunto 4: $177,9 \mathrm{~mm}$ e

- Estação Total: 238,3mm.

Para verificar se a altura das bases travadas se mantém ao longo de diversas calagens, foram realizadas observações em um colimador (figura 6).

Figura 6 - Procedimento para observação dos ângulos verticais da estação total

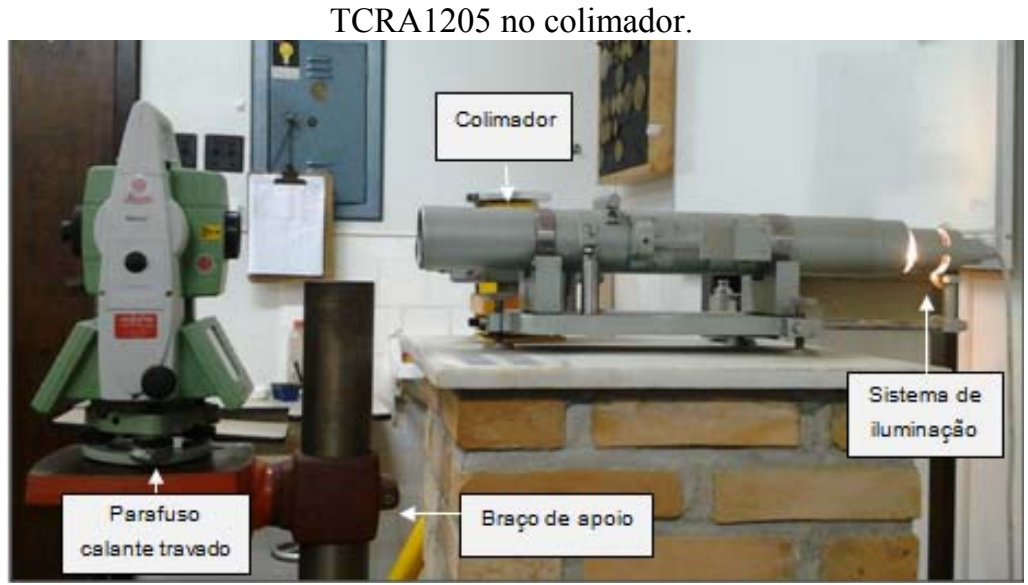

A metodologia consistiu em:

1) Primeiramente instalou-se a estação total em um braço de apoio existente no Laboratório de Instrumentação Geodésica (LAIG), fazendo-se a calagem da mesma; 2) Em seguida, realizaram-se quatro séries de observações em pontaria direta e inversa da luneta;

3) Retirou-se o equipamento do braço de apoio e desconfigurou-se a calagem anterior;

Bol. Ciênc. Geod., sec. Artigos, Curitiba, v. 19, nº 3, p.391-406, jul-set, 2013. 
d) Por fim, instalou-se novamente o equipamento no braço de apoio e repetiu-se os itens 1,2 e 3 mais duas vezes (teste 2 e 3 ).

Avaliando-se estatisticamente os resultados através do teste t de Student para verificar a igualdade entre duas médias, foi possível concluir que os ângulos verticais dos testes 2 e 3 são estatisticamente iguais ao ângulo determinado no teste 1 , ao nível de significância de $5 \%, \log$, o procedimento adotado permite manter a altura dos equipamentos ao longo de diversas calagens.

\section{CAMPANHAS DE MONITORAMENTO NA UHE MAUÁ}

As campanhas de monitoramento foram conduzidas em épocas distintas, a saber: 31/03; 20/04 e 07/06 antes e 18/07; 16/08; 04/09 e 08/11 durante a formação do reservatório.

\subsection{Materialização dos Pontos de Monitoramento}

Antes de efetuar o procedimento de irradiação nos pontos de monitoramento foi necessário instalar prismas do modelo GPR112 (figura 7) a jusante da estrutura, de forma que ficassem espaçados por todo o barramento.

Figura 7 - Prisma GPR112 no muro da barragem.

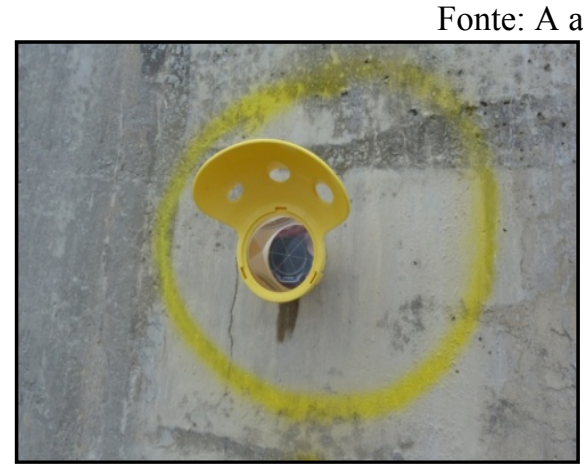

a) Modelo do Prisma

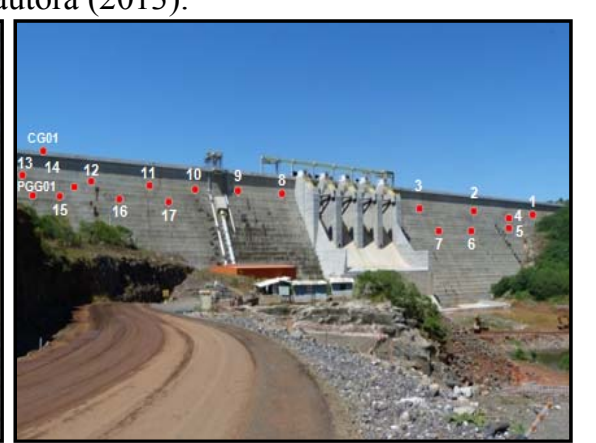

b) Disposição na Barragem

\subsection{Irradiação Tridimensional}

O procedimento adotado para obter as direções horizontais, ângulos verticais e as distâncias inclinadas de todos os pontos de monitoramento a jusante, consistiu em instalar o equipamento (estação Total TCR1205) no pilar geodésico PG03 e realizar a orientação inicial (ré) no pilar PG02, e posteriormente efetuar as irradiações dos prismas a jusante. Como o instrumento utilizado é uma estação robotizada foram feitos no mínimo nove séries de observações em cada um dos prismas, em pontaria direta e inversa da luneta (PD, PI). Todas as informações foram gravadas automaticamente pelo equipamento. A figura 9 ilustra as visadas efetuadas. 
Ressalta-se que como a estação total e o conjunto de base e prisma possuem alturas fixas não é necessário medir estas grandezas em campo.

A estação total utilizada (TCRA1205) realiza leituras de alta precisão, apoiada pela função de reconhecimento automático de alvos $\left(\mathrm{ATR}^{\circledR}\right)$ e pelo sistema de detecção PowerSearch (PS ${ }^{\circledR}$ ), permitindo maior rapidez e menor influência do operador. As principais características técnicas são: precisão da medida angular igual a $5 "$ e na medida da distância igual $\pm(2 \mathrm{~mm}+2 \mathrm{ppm})\left(\right.$ LEICA GEOSYSTEMS $\left.^{\circledR}\right)$.

Figura 9 - Levantamento pelo método de irradiação nos alvos a jusante. Fonte: A autora (2013).

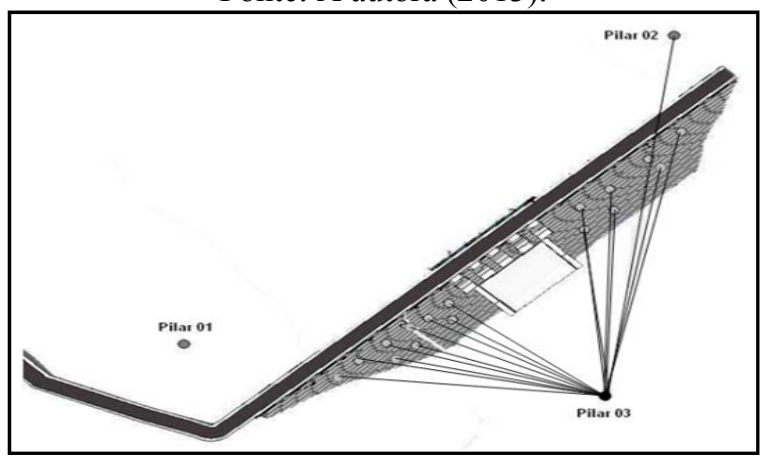

Com intuito de efetuar correções ambientais nas distâncias medidas com a estação total, foram anotadas em cadernetas de campo, em cada uma das séries de observações, ou seja, durante todo o processo de irradiação, os valores da pressão atmosférica, temperatura e umidade relativa do ar. Para que estas informações fossem obtidas com rigor foram utilizados um termo higrômetro digital e um barômetro. Este, a cada campanha de levantamento foi calibrado através da comparação com uma coluna de mercúrio. Rüeger (1996), afirma que as variações nas condições atmosféricas causam um aumento ou diminuição na velocidade de propagação da onda eletromagnética e provocam erros sistemáticos nas medidas das distâncias. Logo, foram efetuadas correções nos dados medidos em campo empregando-se a formulação que consta no manual da estação total utilizada (equação 4). Estas correções foram aplicadas nas distâncias posteriormente, através de um algoritmo computacional desenvolvido.

$$
\Delta D_{1}=283,04-\left[\frac{0,29195 p}{1+\alpha t}-\frac{4,126 \cdot 10^{-4} h}{1+\alpha t} \cdot 10^{x}\right]
$$

onde:

$\Delta D_{1}$ : Correção atmosférica em ppm;

$p$ : pressão atmosférica em mbar;

Bol. Ciênc. Geod., sec. Artigos, Curitiba, v. 19, nº 3, p.391-406, jul-set, 2013. 
$t:$ temperatura do ar ambiente $\mathrm{em}{ }^{\circ} \mathrm{C}$;

$\alpha: 1 / 273,16$;

$h:$ umidade relativa do ar em porcentagem; e $x=\left(\frac{7,5 t}{237,3+t}\right)+0,7857$.

\subsection{Cálculo das Coordenadas Tridimensionais}

A cada campanha de levantamento as leituras das direções horizontais, dos ângulos verticais, bem como das distâncias inclinadas foram gravadas no instrumento utilizado. Estas informações geram um arquivo específico para ser usado no aplicativo denominado Coord_3D, desenvolvido especialmente para as atividades realizadas na UHE Mauá. Devido ao grande volume de dados, ao número de campanhas de levantamento e as inerentes análises adotadas no processo de cálculo das coordenadas tridimensionais, implementou-se na rotina Coord_3D análises estatísticas e também as correções ambientais das distancias.

\section{RESULTADOS}

As coordenadas tridimensionais obtidas na primeira campanha de levantamento, calculadas a partir do pilar geodésico PG03, são tomadas como sendo de referência para os cálculos das diferenças entre as demais épocas. A variação máxima tolerável de deslocamentos verticais (recalque) e horizontais adotados são de 10 e 15 milímetros respectivamente. Estes valores foram definidos por semelhança aos limites adotados na barragem da usina hidrelétrica de Salto Caxias, cujos valores são encontrados no documento intitulado "Instrumentos de Auscultação das obras civis, valores de controle para leituras dos instrumentos" elaborado pela Companhia Paranaense de Energia (COPEL, 1998).

O comportamento das variações das coordenadas obtidas entre a época 1 (referência), calculadas a partir do pilar geodésico PG03 e as demais épocas de levantamento, podem ser visualizadas nas figuras 10,11 e 12 . Como não houve observações para três pontos de monitoramento (MGE_01, MGE_02 e MGE_14) na primeira campanha, tomou-se como referência a época 2 para a comparação apenas destas coordenadas. É importante salientar que o método de monitoramento utilizado não foi comparado com nenhum outro método geralmente empregado no monitoramento de barragens, sendo a validação dos resultados dada a partir dos valores limites estabelecidos.

Analisando-se as variações das coordenadas leste, através das diferenças calculadas entre as campanhas (Tabela 1), é possível verificar que em alguns pontos a variação mínima chega à ordem do centésimo do milímetro $(0,01 \mathrm{~mm})$, enquanto na variação máxima a ordem é centimétrica $(1,42 \mathrm{~cm})$. 
Figura 10 - Variações das coordenadas leste de todas as épocas de levantamentos obtidas por PG03.

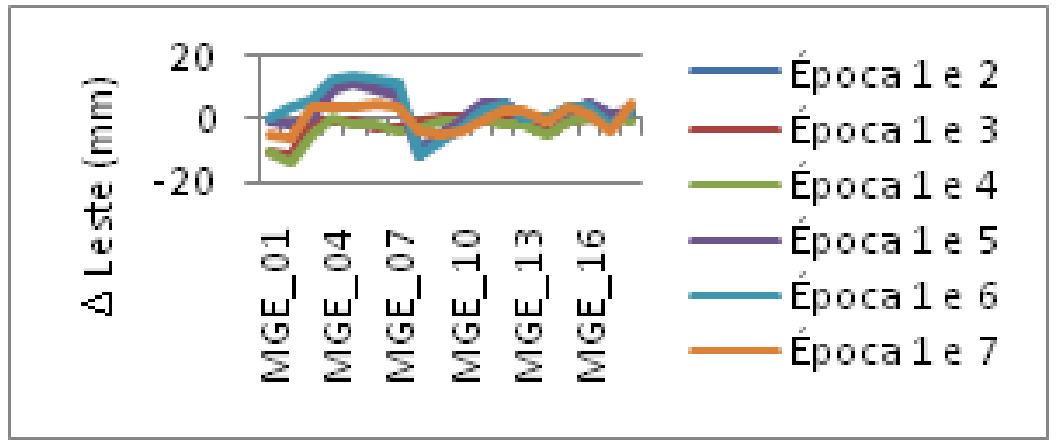

Tabela 1 - Variações das coordenadas leste entre as épocas 1 e 2, 1 e 3, 1 e 4, 1 e 5 ,

\begin{tabular}{|c|c|c|c|c|c|c|}
\hline \multirow{2}{*}{ PG03 } & $\begin{array}{c}\Delta \mathrm{X} \\
(\mathrm{mm})\end{array}$ & $\begin{array}{c}\Delta \mathrm{X} \\
(\mathrm{mm})\end{array}$ & $\begin{array}{c}\Delta \mathrm{X} \\
(\mathrm{mm})\end{array}$ & $\begin{array}{c}\Delta \mathrm{X} \\
(\mathrm{mm})\end{array}$ & $\begin{array}{c}\Delta \mathrm{X} \\
(\mathrm{mm})\end{array}$ & $\begin{array}{c}\Delta \mathrm{X} \\
(\mathrm{mm})\end{array}$ \\
\hline & $\begin{array}{c}\text { Época } \\
1 \text { e } 2\end{array}$ & $\begin{array}{c}\text { Época } \\
1 \text { e } 3\end{array}$ & $\begin{array}{c}\text { Época } \\
1 \text { e } 4\end{array}$ & $\begin{array}{c}\text { Época } \\
1 \text { e } 5\end{array}$ & $\begin{array}{l}\text { Época } \\
1 \text { e } 6\end{array}$ & $\begin{array}{c}\text { Época } \\
1 \text { e } 7\end{array}$ \\
\hline MGE_01 & & $-11,4$ & $-11,0$ & $-0,8$ & 1,4 & $-5,0$ \\
\hline MGE_02 & & $-12,1$ & $-13,9$ & $-1,4$ & 3,8 & $-5,7$ \\
\hline MGE_03 & 3,1 & $-1,1$ & $-5,5$ & 0,4 & 6,2 & 3,2 \\
\hline MGE_04 & 11,5 & $-1,6$ & $-0,2$ & 9,7 & 13,1 & 3,8 \\
\hline MGE_05 & 12,2 & $-1,1$ & $-1,4$ & 11,4 & 14,2 & 3,3 \\
\hline MGE_06 & 10,8 & $-3,2$ & $-2,1$ & 9,2 & 13,6 & 4,4 \\
\hline MGE_07 & 8,8 & $-2,8$ & $-4,1$ & 6,7 & 11,8 & 3,2 \\
\hline MGE_08 & $-9,2$ & $-1,2$ & $-3,2$ & $-11,7$ & $-11,4$ & $-3,4$ \\
\hline MGE_09 & $-4,7$ & $-0,5$ & $-1,3$ & $-5,6$ & $-7,5$ & $-4,8$ \\
\hline MGE_10 & 0,8 & 0,0 & $-0,7$ & $-1,0$ & $-2,5$ & $-3,5$ \\
\hline MGE_11 & 4,6 & $-0,6$ & 0,3 & 4,4 & 3,2 & $-0,3$ \\
\hline MGE_12 & 4,8 & $-0,7$ & $-1,5$ & 5,2 & 5,6 & 2,1 \\
\hline MGE_13 & $-0,5$ & $-1,2$ & $-1,7$ & 0,0 & 0,9 & 2,4 \\
\hline MGE_14 & & $-4,8$ & $-4,7$ & $-0,1$ & 1,2 & $-1,1$ \\
\hline MGE_15 & 2,5 & $-1,1$ & $-1,3$ & 2,1 & 3,8 & 2,9 \\
\hline MGE_16 & 4,6 & $-0,4$ & 0,1 & 4,6 & 4,6 & 1,3 \\
\hline MGE_17 & 1,9 & $-0,9$ & 0,2 & 1,1 & $-1,1$ & $-3,3$ \\
\hline PGG01 & 1,6 & & $-0,7$ & 2,3 & 2,6 & 3,9 \\
\hline
\end{tabular}

Bol. Ciênc. Geod., sec. Artigos, Curitiba, v. 19, nº 3, p.391-406, jul-set, 2013. 
Figura 11 - Variações das coordenadas norte de todas as épocas de levantamentos obtidas por PG03.

Fonte: A autora (2013).

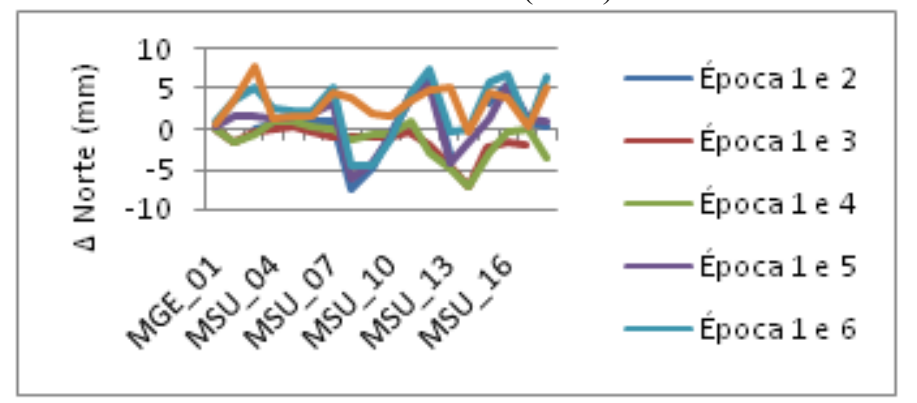

Já na comparação entre as coordenadas norte (Tabela 2), as máximas e as mínimas variações são de $7,5 \mathrm{~mm}$ e $0,01 \mathrm{~mm}$ respectivamente.

Tabela 2 - Variações das coordenadas norte entre as épocas 1 e 2, 1 e 3, 1 e 4, 1 e 5,

\begin{tabular}{l|c|c|c|c|c|c}
\hline \multirow{3}{*}{ PG03 } & $\Delta \mathrm{Y}(\mathrm{mm})$ & $\Delta \mathrm{Y}(\mathrm{mm})$ & $\Delta \mathrm{Y}(\mathrm{mm})$ & $\Delta \mathrm{Y}(\mathrm{mm})$ & $\Delta \mathrm{Y}(\mathrm{mm})$ & $\Delta \mathrm{Y}(\mathrm{mm})$ \\
\cline { 2 - 7 } & $\begin{array}{c}\text { Época } \\
1 \mathrm{e} 2\end{array}$ & $\begin{array}{c}\text { Época } \\
1 \mathrm{e} 3\end{array}$ & $\begin{array}{c}\text { Época } \\
1 \mathrm{e} 4\end{array}$ & $\begin{array}{c}\text { Época } \\
1 \text { e } 5\end{array}$ & $\begin{array}{c}\text { Época } \\
1 \text { e 6 }\end{array}$ & $\begin{array}{c}\text { Época } \\
1 \mathrm{e} 7\end{array}$ \\
\hline MGE_01 & & $-0,1$ & $-0,3$ & 0,4 & 0,9 & 0,8 \\
\hline MGE_02 & & $-1,5$ & $-1,8$ & 1,6 & 3,4 & 3,8 \\
\hline MGE_03 & $-0,1$ & $-0,5$ & $-0,9$ & 1,8 & 5,3 & $-4,4$ \\
\hline MGE_04 & 0,8 & 0,0 & 0,7 & 1,3 & 2,4 & $-3,6$ \\
\hline MGE_05 & 0,8 & 0,3 & 0,8 & 1,7 & 2,2 & $-2,2$ \\
\hline MGE_06 & 1,1 & $-0,3$ & 0,1 & 1,9 & 2,3 & $-2,9$ \\
\hline MGE_07 & 1,0 & $-0,9$ & $-0,3$ & 3,2 & 5,2 & $-2,7$ \\
\hline MGE_08 & $-7,4$ & $-0,9$ & $-1,4$ & $-6,1$ & $-4,6$ & $-3,0$ \\
\hline MGE_09 & $-4,8$ & $-1,1$ & $-0,9$ & $-4,1$ & $-4,7$ & $-2,6$ \\
\hline MGE_10 & $-0,8$ & $-1,1$ & $-0,8$ & $-0,8$ & $-1,4$ & $-2,7$ \\
\hline MGE_11 & 3,9 & $-0,4$ & 0,8 & 4,5 & 4,4 & $-1,9$ \\
\hline MGE_12 & 6,2 & $-1,9$ & $-2,9$ & 5,5 & 7,5 & $-1,7$ \\
\hline MGE_13 & $-3,2$ & $-4,2$ & $-5,0$ & $-4,1$ & $-0,3$ & $-1,1$ \\
\hline MGE_14 & & $-7,1$ & $-7,1$ & $-1,4$ & 2,1 & $-0,2$ \\
\hline MGE_15 & 3,3 & $-2,2$ & $-3,0$ & 1,4 & 5,7 & $-1,6$ \\
\hline MGE_16 & 5,5 & $-1,5$ & $-0,6$ & 5,6 & 6,7 & $-1,4$ \\
\hline MGE_17 & 1,1 & $-2,0$ & 0,0 & 1,4 & 0,3 & $-2,6$ \\
\hline PGG01 & 0,2 & & $-3,6$ & 1,0 & 6,4 & $-3,1$ \\
\hline
\end{tabular}

Bol. Ciênc. Geod., sec. Artigos, Curitiba, v. 19, nº 3, p.391-406, jul-set, 2013. 
Figura 12 - Variações das coordenadas altimétricas de todas as épocas de levantamentos obtidas por PG03.

Fonte: A autora (2013).

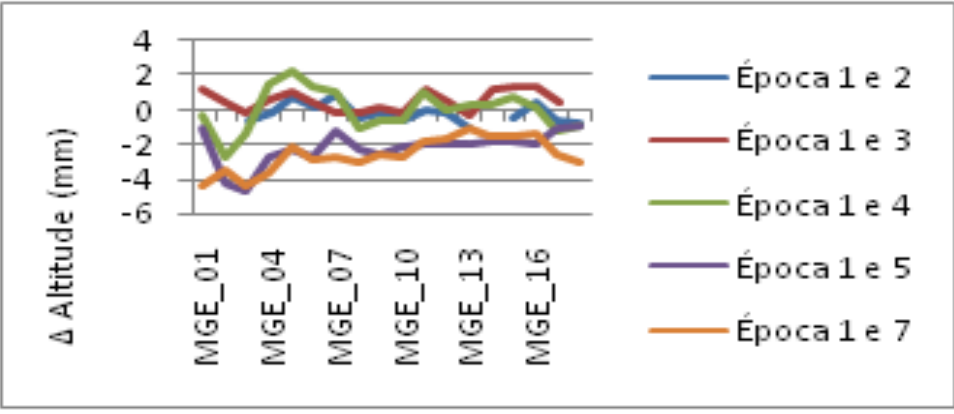

Finalmente, a máxima e a mínima variação altimétrica detectada na comparação entre a época de referência e as demais campanhas de levantamentos (Tabela 3) são de $4,7 \mathrm{~mm}$ e $0,1 \mathrm{~mm}$ respectivamente.

Tabela 3 - Variações das coordenadas altimétricas entre as épocas 1 e 2, 1 e 3, 1 e 4,1 e 5,1 e 6 , e 1 e 7 a partir de PG03

\begin{tabular}{c|c|c|c|c|c|c}
\hline \multirow{2}{*}{ PG03 } & $\Delta \mathrm{Z}(\mathrm{mm})$ & $\Delta \mathrm{Z}(\mathrm{mm})$ & $\Delta \mathrm{Z}(\mathrm{mm})$ & $\Delta \mathrm{Z}(\mathrm{mm})$ & $\Delta \mathrm{Z}(\mathrm{mm})$ & $\Delta \mathrm{Z}(\mathrm{mm})$ \\
\cline { 2 - 7 } & $\begin{array}{c}\text { Época } \\
1 \text { e 2 }\end{array}$ & $\begin{array}{c}\text { Época } \\
1 \text { e 3 }\end{array}$ & $\begin{array}{c}\text { Época } \\
1 \text { e 4 }\end{array}$ & $\begin{array}{c}\text { Época } \\
1 \text { e 5 }\end{array}$ & $\begin{array}{c}\text { Época } \\
1 \text { e 6 }\end{array}$ & $\begin{array}{c}\text { Época } \\
1 \text { e 7 }\end{array}$ \\
\hline MGE_01 & & 1,1 & $-0,3$ & $-1,1$ & $-2,2$ & $-4,4$ \\
\hline MGE_02 & & 0,4 & $-2,8$ & $-4,3$ & $-4,0$ & $-3,5$ \\
\hline MGE_03 & $-0,7$ & $-0,1$ & $-1,4$ & $-4,7$ & $-4,0$ & $-4,4$ \\
\hline MGE_04 & $-0,2$ & 0,6 & 1,5 & $-2,7$ & $-1,5$ & $-3,6$ \\
\hline MGE_05 & 0,7 & 1,0 & 2,3 & $-2,2$ & $-1,7$ & $-2,2$ \\
\hline MGE_06 & 0,1 & 0,4 & 1,3 & $-2,8$ & $-3,2$ & $-2,9$ \\
\hline MGE_07 & 0,9 & $-0,1$ & 1,1 & $-1,2$ & $-1,7$ & $-2,7$ \\
\hline MGE_08 & $-0,5$ & $-0,1$ & $-1,1$ & $-2,2$ & $-2,7$ & $-3,0$ \\
\hline MGE_09 & $-0,2$ & 0,2 & $-0,6$ & $-2,6$ & $-3,0$ & $-2,6$ \\
\hline MGE_10 & $-0,7$ & $-0,2$ & $-0,6$ & $-2,1$ & $-3,3$ & $-2,7$ \\
\hline MGE_11 & 0,0 & 1,2 & 1,1 & $-2,0$ & $-1,5$ & $-1,9$ \\
\hline MGE_12 & $-0,1$ & 0,4 & 0 & $-2,0$ & $-2,1$ & $-1,7$ \\
\hline MGE_13 & $-1,1$ & $-0,3$ & 0,3 & $-2,0$ & $-1,8$ & $-1,1$ \\
\hline MGE_14 & & 1,1 & 0,3 & $-1,8$ & $-1,0$ & $-1,5$ \\
\hline MGE_15 & $-0,5$ & 1,3 & 0,8 & $-1,8$ & $-0,8$ & $-1,6$ \\
\hline MGE_16 & 0,5 & 1,3 & 0,1 & -2 & $-0,9$ & $-1,4$ \\
\hline MGE_17 & $-0,6$ & 0,5 & $-1,2$ & $-1,1$ & $-1,9$ & $-2,6$ \\
\hline PGG01 & $-0,8$ & & $-0,9$ & $-0,9$ & $-2,9$ & $-3,1$ \\
\hline
\end{tabular}

Bol. Ciênc. Geod., sec. Artigos, Curitiba, v. 19, nº 3, p.391-406, jul-set, 2013. 


\section{CONCLUSÃO}

No âmbito dos levantamentos geodésicos, a execução do método de irradiação tridimensional, por meio de uma estação total robotizada, tornou o monitoramento absoluto dos dezessete prismas instalados na face de jusante da barragem, mais ágil e eficiente.

$\mathrm{O}$ instrumental utilizado é de fácil operação e melhora o rendimento em campo, pois minimiza a influência do observador e acaba por melhorar a precisão das observações. No entanto, é importante atentar para alguns erros que podem atingir os dados observados, como a refração atmosférica. Logo, recomenda-se que sejam efetuados estudos para determinar qual a influência da refração atmosférica nas medidas e como corrigi-las. Uma saída seria efetuar campanhas de levantamentos noturnas, minimizando assim a influência deste fenômeno.

O aplicativo desenvolvido, tornou possível otimizar todos os passos necessários para a obtenção das coordenadas tridimensionais, o que muitas vezes não é possível utilizando softwares comerciais.

Após o cálculo das diferenças das coordenadas dos pontos monitorados entre todas as épocas de levantamento, foi possível constatar que algumas variações chegam à ordem centimétrica, no entanto, nenhum ponto apresentou diferenças entre campanhas acima dos valores admissíveis adotados.

Recomenda-se a continuidade dos estudos realizando-se uma transformação do sistema topográfico local para um sistema da barragem. Um dos eixos seria paralelo a mesma, um ortogonal (sentido do fluxo) e o terceiro descendente para representar o recalque da estrutura.

\section{AGRADECIMENTOS:}

Ao projeto de pesquisa: "Levantamento Geológico-Estrutural da Região e Entorno da Barragem da UHE Mauá, uma Abordagem Inovadora para o Monitoramento Geodésico nos Pontos de Risco" financiado pela ANEEL/COPEL.

\section{REFERÊNCIAS BIBLIOGRÁFICAS}

BRASIL. Ministério da Integração Nacional. Manual de segurança e inspeção de barragens. Brasília: MIN, 2002. Disponível em: <http://www.mi.gov.br/ infraestruturahidrica/publicacoes/manual_barragens.asp $>$. Acesso em:05/05/ 2012.

CASTRO A. T; HENRIQUES M. J. Monitoring planimetric displacements in concrete dams. $13^{\circ} \mathrm{FIG}$ - Simpósio sobre análises e medidas de deformação. $4^{\circ}$ IAG - Simpósio sobre geodésia para engenharia geotécnica e estrutural. Lisboa: 2008. Disponível em: <http://www.fig.net/commission6/lisbon 2008/papers/pas05/ pas05_02_tavaresdecastro_mc127.pdf>. Acesso em: $15 / 05 / 2012$.

CHRZANOWSKI, A. Geotechnical and other non-geodetic method in deformation measurement. 1986, In: Proceedings of the Deformation Measurement 
Workshop. Oct 31 - Nov 1,Ed: Yehuda Bock, Massachusetts Institute of Technology, Cambridge, MA.pp.112-152.

CONSÓRCIO ENERGÉTICO CRUZEIRO DO SUL. Usina Hidrelétrica Mauá. Disponível em: <http://www.usinamaua.com.br/>. Acesso em: 05/04/2012.

COPEL. COMPANHIA PARANAENSE DE ENERGIA. Usina Hidrelétrica de Salto Caxias. Instrumentos de auscultação das obras civis, valores de controle para leituras dos instrumentos. Novembro de 1998.

DEPARTMENT OF ARMY - U. S. ARMY CORPS OF ENGENEERS.Manual 1110- 1-1004. Deformation monitoring and control surveying. Washington, DC, 1994. Obtido em:<http://gisceu.net/PDF/u44.pdf $>$. Acesso em: 07/05/2012.

LEICA GEOSYSTEMS ${ }^{\circledR}$. TPS1200. Manual de operação. Versão 1.0, Português.

MIRANDA, F. A., FAGGION P. L., VEIGA, L. A. Método para o monitoramento de pontos em áreas instáveis. Boletim de Ciências Geodésicas, sec. Artigos, Curitiba, v. 15, n 4, p.498-513, outubro a dezembro, 2009.

NADAL, C. A., VEIGA, L. A. K., FAGGION, P. L., FREITAS, S. R. C. DE,ZOCOLOTTI FILHO, C. A., GRANEMANN, D. C., LOPES, C. R., SANTOS, D. P. DOS. Integração da Auscultação Geodésica com a Instrumentação de Controle e Segurança da Barragem de Salto Caxias. Relatório Técnico, Universidade Federal do Paraná, 2004.

RÜEGER, J. M. Electronic Distance Measurement: an introduction. Ed 3. Berlin: Springer Verlag, 1996.

(Recebido em abril de 2013. Aceito em junho de 2013). 